\title{
Dermatophytosis Caused by Microsporum canis in a Free-Living Maned Wolf (Chrysocyon brachyurus)
}

\author{
Keylla Helena Nobre Pacifico Pereira', Elton Luís Ritir Oliveira ${ }^{2}$, Raphael Augusto Baldissera Gonçalves ${ }^{2}$, \\ Luna Scarpari Rolim², Ramiro das Neves Dias Neto ${ }^{3}$, Maíra Sales Castilho ${ }^{4}$, \\ Carlos Roberto Teixeira ${ }^{4} \&$ Sheila Canavese Rahal ${ }^{4}$
}

\begin{abstract}
Background: Chrysocyon brachyurus is a South American wild canid considered a species near threatened by the International Union for Conservation of Nature and is classified as vulnerable in the National List of Endangered Species. With the increase of the contact between domestic animals, human population and wild animals, there was a greater exposure of the maned wolf to pathogens. Due to the importance of its conservation, the knowledge of emerging infectious diseases that affect this species becomes essential. This report aims to describe the first diagnosed case of dermatophytosis caused by Microsporum canis in a maned wolf.

Case: A free-living female maned wolf (Chrysocyon brachyurus), approximately 60 days old, was rescued with presence of alopecia, non-pruritic lesions, one of circular shape located in the nasal plane and the other with signs of scaling and crusts in the region of the left pina. The animal was active, in good general condition and without other significant changes to clinical examination. Skin scraping was performed for mycological culture of both lesions. Fungal growth on Sabouraud's agar identified Microsporum canis. Topical therapy with ketoconazole ointment and cleaning of lesions with $0.2 \%$ chlorhexidine was instituted. After 20 days of treatment, remission of clinical signs and repilation of affected areas were observed. New mycological cultures of both areas were carried out, which were negative for Microsporum canis. Discussion: Although Microsporum canis is described as causing dermatophytosis in several animal species, it has apparently not yet been reported in maned wolves. Microsporum canis is one of the most isolated zoophilic dermatophytes in domestic cats and is also cited in reports of symptomatic wild felids, such as tigers, in which it has been identified as either the only agent of infection or in association with Trichophyton mentagrophytes. The occurrence is also high in domestic dogs. In the wild canids, Microsporum gypseum has been described in the gray wolf, Trichophyton mentagrophytes in wild foxes, and Microsporum canis in red fox, among others. The finding of zoophilic and geophilic dermatophyte fungi in healthy, free-living and captive wild animals confirms their role as carriers, probable reservoirs and disseminators of these organisms in the environment, and the potential risk they represent as causes of zoonoses. Zoophilic dermatophytes are usually transmitted by contact between individuals and contaminated fomites. In the case of $M$. canis in the red fox the contact was an asymptomatic domestic cat. In the present case the transmission was not determined, since the animal was rescued from the wild. Regarding the age group, young animals are more susceptible to dermatophytes than adults, probably related to the greater immunity of adults due to previous contacts or even the immaturity of the immune system of the young. The dermatophytosis diagnosis is generally based on anamnesis, physical examination of the lesion, Wood's lamp examination, microscopic skin scraping, fungal culture, or even histology and PCR tests. In the present case, the final diagnosis was based upon by fungal culture in Sabouraud agar, which allowed to identify the dermatophyte species and, thus, the possible source of infection. Dermatophyte infections can be treated with systemic or topical antifungal medications. Because it was a young animal, it was chosen in the present case only for topical use, which proved to be adequate, probably due to the action of the drug in the areas of alopecia skin and the improvement of the systemic condition of the animal. Knowing that the maned wolf is considered as a vulnerable species, it is important to know the diseases that affect this species, in order to carry out, when necessary, disease monitoring programs, preventive and therapies, which is essential for its preservation.
\end{abstract}

Keywords: wild animal, disease, skin, dermatophyte.

Received: 30 0ctober 2017

Accepted: 4 February 2018

Published: 16 February 2018

${ }^{1}$ Programa de pós-graduação em Clínica Veterinária \& ${ }^{2}$ Residência em Clínica e Cirurgia de Animais Selvagens, Faculdade de Medicina Veterinária e Zootecnia - UNESP, Botucatu, SP, Brazil. ${ }^{3}$ M.V. do Zoológico do Rio de Janeiro (RioZoo), Rio de Janeiro, Brazil. ${ }^{4}$ Departamento de Cirurgia Veterinária e Anestesiologia, Faculdade de Medicina Veterinária e Zootecnia - UNESP, Botucatu. CORRESPONDENCE: K.H.N.P. Pereira [keylla_pacifico@ hotmail. com - Tel.: +55 (14) 3880-2039]. Universidade Estadual Paulista (UNESP). Rubião Júnior s/n. CEP 18618970 Botucatu, SP, Brazil. 


\section{INTRODUCTION}

The anthropogenic impact on the environment has destroyed the natural habitat of wild animals that favors occurrence of diseases caused by agents from domestic animals in the wild populations [4]. Among these diseases is the dermatophytosis, which is a contagious disease caused by fungi belonging to the genera Microsporum, Trichophyton and Epidermophyton [12,13]. However, only members of the genera Microsporum and Trichophyton produce lesions in animals $[2,4]$.

The dermatophytes are capable to break down keratin in tissues including epidermis, hair and nails $[4,9,16]$. Fungus infections occur by the transmission of fungal propagules to a susceptible host $[4,16]$.

The domestic mammals are susceptible to dermatophytes, as well as wild mammals $[3,6]$. In wild animals, the dermatophytosis has been described in several species of asymptomatic and symptomatic felids $[1,3,6,14,17,18]$, but less frequently in canids $[7,10,11]$. In canids there are reports dealing with wild fox [10], red fox, [11], gray wolf [7], among others.

The maned wolf (Chrysocyon brachyurus) is considered as the largest canid in South America, which is observed in Brazil, Bolivia and in a small region in eastern of Peru, Paraguay, Argentina, and Uruguay [15]. In Brazil, the maned wolf can be found from northeast region to a portion of Rio Grande do Sul state; including areas of Pantanal, Cerrado and Caatinga, as well as Atlantic Forest areas [5]. The species has been categorized as Near Threatened by the IUCN Red List [15],

The purpose of this report is to describe the clinical features, diagnosis and treatment of dermatophyte infection in a free-living maned wolf.

\section{CASE}

A female free-ranging maned wolf (Chrysocyon brachyurus), approximately 60 days old, and weighing $2.55 \mathrm{~kg}$ was referred to the Center of Medicine and Research in Wild Animals (CEMPAS) after being rescued by Environmental police. Physical examination showed two areas of nonpruritic alopecia, one circular lesion was located on the muzzle $(4.5 \mathrm{~cm}$ in diameter) and another with signs of scaling and crusting on the left pinna ( $6.5 \mathrm{~cm}$ in length) [Figure 1]. The animal was alert, the body condition was very good, and had no other signs of illness on physical examination.
Skin scale of both lesions were collected for mycological culture. The samples were cultured on Sabouraud agar, and incubated at room temperature for 14 days. Samples of the colonies were then stained with methylene blue and examined microscopically. Based on micromorphological characteristics of colonies that contained fusiform macroconidia with 6 or more cells, thick walls and septate hyphae, the diagnosis was $M i$ crosporum canis. Topical therapy using ketoconazole cream $^{1}(20 \mathrm{mg} / \mathrm{g})$ and cleaning of the skin lesions with $0.2 \%$ chlorhexidine ${ }^{2}$ was used. Remission of clinical signs and hair growth in the affected areas were observed 20 days after therapy (Figure 2). Samples of both areas were taken again and were negative on mycological culture.

\section{DISCUSSION}

The anthropogenic impact on the environment has destroyed the natural habitat of wild animals that favors occurrence of diseases caused by agents from domestic animals in the wild populations [4]. Among these diseases is the dermatophytosis, which is a contagious disease caused by fungi belonging to the genera Microsporum, Trichophyton and Epidermophyton [11]. However, only members of the genera Microsporum and Trichophyton produce lesions in animals [2,4], as observed in the present case.

The wild mammals are susceptible to dermatophytes, as well as domestic mammals [3]. In wild animals, the dermatophytosis has been described in several species of asymptomatic and symptomatic felids [3,14,17,18], but less frequently in canids $[7,10,11]$. In canids may be cited reports in wild fox [10], red fox [11], and gray wolf [7], among others. To the authors' knowledge, this is the first case reported in maned wolf. The maned wolf (Chrysocyon brachyurus) is considered the largest canid in South America, which is observed in Brazil, Bolivia and in a small region in eastern of Peru, Paraguay, Argentina, and Uruguay [15]. The species has been categorized as Near Threatened by the IUCN Red List [15].

In animals, dermatophytosis is characterized by single or multiple areas of alopecia, localized in any part of the body $[2,12,16]$. Generally, the lesions take on a rough circular appearance, with presence of scaling, crusts and erythema, and may or may not be pruritic $[2,9,12,16]$. In the present case, the lesion on the muzzle was more characteristic than the lesion on the ear, although in both Microsporum canis has been isolated. 


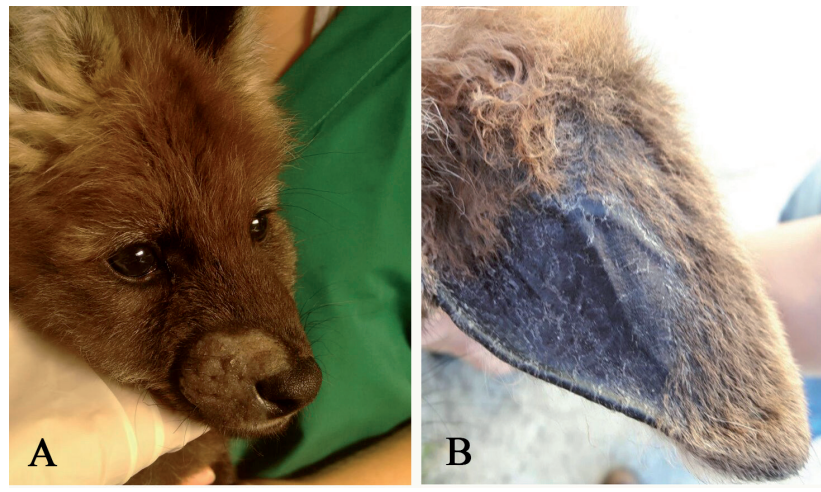

Figure 1. Areas of nonpruritic alopecia, one circular lesion located on the muzzle (A) and another with signs of scaling and crusting on the left ear (B) in a young maned wolf (Chrysocyon brachyurus), suggesting dermatophytosis.
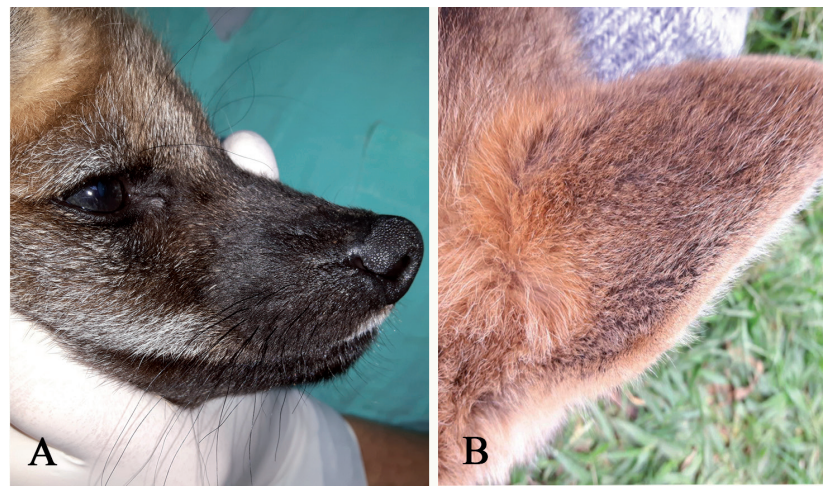

Figure 2. Regression of the dermatophyte lesions located on the muzzle area (A) and left ear (B), after 20 days of topic treatment with antifungal medications in a young maned wolf (Chrysocyon brachyurus).

The diagnosis of dermatophytosis is, in general, based on anamnesis, physical examination, and microscopic examination of skin scraping and hair plucking of the lesions, which should be confirmed by Wood's lamp examination, direct examination, fungal culture, or even histological examination and PCR tests $[2,4,12]$. In the present case, the diagnosis was by fungal growth on Sabouraud's agar, which allowed the identification of dermatophyte species and, consequently, the possible source of infection. The prevalence of different dermatophyte species is related to temperature, climate, humidity of geographic regions, and presence of reservoirs $[2,12]$.

Microsporum canis is the most isolated zoophilic dermatophytes from domestic cats [2]. It also has been reported in symptomatic wild felids, such as tigers, which was identified as unique isolated [17,18], or in association to Trichophyton mentagrophytes [14]. In wild canids, Microsporum gypseum has been described in a gray wolf [7], Trichophyton mentagrophytes in eight captured wild red fox [10], and Microsporum canis in a red fox [11], among others.

Zoophilic dermatophytes are usually transmitted by direct contact with infected host, or infected fomites [2]. In the case of Microsporum canis in a red fox, the contactant was an asymptomatic domestic cat [11]. In the present case, the mode of transmission was not determined, since the animal was free-living.

Dermatophyte infections can be treated with antifungals administered systemically, or topically in the form of creams and shampoos, associated with environmental cleaning $[4,12]$. In dogs and cats, the most appropriate therapy is a combination of systemic and topical antifungal agents, since topical antifungals are considered incapable to eliminate dermatophytes from within hair or hair follicles $[9,12,16]$. Because the patient was a young maned wolf, only topical antifungal therapy was used. This was proved adequate, probably due to the action of antifungal in the affected areas with hair loss, and by an improvement in the general condition of the animal. In addition, healthy animals may have heal spontaneous of the lesions within one to a few months [8].

Young animals are, in general, more susceptible to dermatophytes than adults, probably due to the greater immunity of adults related to a previous contact, or even by the immaturity of the immune system in young $[2,4,9]$. However, should be considered that Microsporum canis induces low-grade immune response [2]. Although the animal in the present report is considered young (approximately 60 days of age), other factors such as animal health status and preexisting diseases, may influence the establishment of infections $[2,12]$.

Based on characteristic lesions and mycological culture was possible to conclude that maned wolf can be infected by Microsporum canis dermatophyte that can be topically treated.

\section{MANUFACTURERS}

${ }^{1}$ Janssen-Cilag Farmacêutica Ltda. São José dos Campos, SP, Brazil. ${ }^{2}$ Rioquímica. São José do Rio Preto, SP, Brazil.

Declaration of interest. The authors report no conflicts of interest. The authors alone are responsible for the content and writing of the paper. 


\section{REFERENCES}

1 Bentubo H.D.L., Fedullo J.D., Corrêa S.H.R., Teixeira R.H.F. \& Coutinho S.D. 2006. Isolation of Microsporum gypseum from the haircoat of health wild felids kept in captivity in Brazil. Brazilian Journal of Microbiology. 37(2): 148-152.

2 Chermette R., Ferreiro L. \& Guillot J. 2008. Dermatophytoses in animals. Mycopathologia. 166(5-6): 385-405.

3 Costa E.O., Diniz L.S.M., Carvalho V.M., Coutinho S. \& Benites N.R. 1995. Dermatoses observed in man, laboratory, domestic and wild animals, in Sao Paulo, Brazil. Restrospective study. Arquivo Brasileiro de Medicina Veterinária e Zootecnia. 47(4): 601-607.

4 Coutinho S.D.A. 2014. Dermatofitoses. In: Cubas Z.S., Silva J.C.R. \& Catão-Dias J.L. (Eds). Tratado de animais selvagens. São Paulo: Roca, pp.1411-1418.

5 Cubas Z.S., Silva J.C.R. \& Catão-dias J.L. 2014. Tratado de animais selvagens. 2.ed. São Paulo: Roca, 765p.

6 Figueiredo M.A.P., Manrique W.G., Belo M.A.A. \& Reis H.R.C. 2014. Zoonotic potential of health wild felids for dermatophytes. Journal of Animal and Veterinary Advances. 13: 1018-1021.

7 Fischman O., Siqueira P.A. \& Baptista G. 1987. Microsporum gypseum infection in a gray wolf (Canis lupus) and a camel (Camelus bactrianus) in a zoological garden. Mycoses. 30(7): 295-297

8 Grappel S.F., Bishop C.T. \& Blank F. 1974. Immunology of dermatophytes and dermatophytosis. Bacteriological Review. 38(2): 222-250.

9 Hnilica K.A. \& Patterson A.P. 2016. Small animal dermatology: A color atlas and therapeutic guide. Saint Louis: Elsevier, 104p.

10 Knudtson W., Gates C., Ruth G. \& Hadley L.D. 1980. Trichophyton mentagrophytes_dermatophyosis in wild fox. Journal of Wildlife Diseases. 16(4): 465-468.

11 Malmasi A., Khosravi A.R., Selk Ghaffari M. \& Shojaee Tabrizi A. 2009. Microsporum canis infection in a red fox (Vulpes vulpes). Iranian Journal of Veterinary Research. 10(2): 189-191.

12 Mattei A.S., Beber M.A. \& Madrid I.M. 2014. Dermatophytosis in small animals. Symbiosis. 2(3): 1-6.

13 Merchant S.R. 2013. Dermatophytosis. The Merck Veterinary Manual Online. [Fonte: http://www.merckmanuals.com/ vet/integumentary_system/dermatophytosis_ringworm/overview_of_der matophytosis.html]. [Accessed online in July 2015].

14 Mihaylov G., Petrov V. \& Zhelev G. 2008. Comparative investigation on several protocols for treatment of dermatophytoses in pets. Trakia Journal of Sciences. 6(1): 102-105.

15 Paula R.C. \& DeMatteo K. 2016. Chrysocyon brachyurus. The IUCN red list of threatened species 2015 [Internet]. e.T4819A88135664. [cited 2016 April 07]. [Fonte:<http://www.iucnredlist.org/details/4819/0>]. [Accessed online in August 2016].

16 Patel A. \& Forsythe P.J. 2008. Saunders solutions in veterinary practice: small animal dermatology. Philadelphia: Saunders Elsevier, pp.169-172.

17 Sykes J.M. \& Ramsay E.C. 2007. Attempted treatment of tigers (Panthera tigris) infected with Microsporum canis. Journal of Zoo and Wildlife Medicine. 38(2): 252-257.

18 Takatori K., Ichijo S. \& Kurata H. 1981. Dermatophytosis of tiger caused by Microsporum canis. Mycopathologia. 73(2): 105-108. 